\title{
Commentary \\ A Potential Role for Oxytocin in the Intergenerational Transmission of Secure Attachment
}

\author{
James K Rilling*, 1,2,3,4 \\ 'Department of Anthropology, Emory University, Atlanta, GA, USA; ${ }^{2}$ Department of Psychiatry and Behavioral Sciences, Emory University School \\ of Medicine, Atlanta, GA, USA; ${ }^{3}$ Center for Behavioral Neuroscience, Emory University, Atlanta, GA, USA; ${ }^{4}$ Division of Psychobiology, Yerkes \\ National Primate Research Center, Emory University, Atlanta, GA, USA
}

Neuropsychopharmacology (2009) 34, 262 I-2622; doi:I0.1038/npp.2009.136; published online 2 September 2009

If a significant amount of psychopathology stems from insecure attachment during infancy and childhood (Berant et al, 2008), then much of this illness is perpetuated by the intergenerational transmission of attachment styles (van Ijzendoorn et al, 1995). One fundamental challenge for psychiatry is to break this cycle, and doing so will require knowledge of the biological and psychosocial mechanisms that fuel it.

In this issue, Strathearn and colleagues present evidence that the neuropeptide oxytocin may be an important component of this mechanism. Oxytocin mediates motherinfant attachment in rats and sheep, as well as adult female pair bond formation in prairie voles (Insel and Young, 2001); hence it is reasonable to hypothesize that it might also mediate attachment between human mothers and their children. Recently, researchers have begun investigating the role of oxytocin in human behavior through the use of intranasal oxitocin sprays that seem to enable neuropeptides to cross the blood-brain barrier (Born et al, 2002). These studies have demonstrated several remarkable behavioral effects of oxytocin in human subjects, including increased gazing to the eye region of faces, improved face expression recognition, enhanced encoding of positive social memories, decreased behavioral and neuroendocrine responses to social stress, increased positive communication between couples, and increased levels of trust and generosity in economic games (Ebstein et al, 2009; Heinrichs and Domes, 2008). Most of these studies have been conducted with male subjects, however, and human maternal behavior has not yet been targeted with this approach.

In this issue, Strathearn and colleagues investigate the influence of the oxytocin system on attachment with an

*Correspondence: Dr JK Rilling, Department of Anthropology, Psychiatry and Behavioral Sciences, Emory University, 1557 Dickey Drive, Atlanta, GA 30322, USA, Tel: + 404727 3062,

Fax: + 404727 2860, E-mail: jrillin@emory.edu

Received 31 July 2009; accepted 31 July 2009 alternative and innovative approach that emphasizes ecological validity. During pregnancy, women were assessed for attachment styles and categorized as either Type A (insecure, dismissive attachment) or Type B (securely attached) mothers. Approximately 7 months after giving birth, the mothers' plasma oxytocin response to an interaction with their infant was measured, and Type B mothers were found to have a greater oxytocin response than Type A mothers. Approximately 11 months after delivery, both types of mothers were imaged with fMRI, as they viewed pictures of both their own and other infants displaying happy, sad, or neutral facial expressions. Type B mothers, who had earlier exhibited the stronger oxytocin response to interactions with their infants, showed a stronger ventral striatum response to their infant's happy and sad faces than did Type A mothers. Moreover, there was a significant positive correlation between a mother's oxytocin response when interacting with her infant and her ventral striatum response when later viewing her infant's face in the MRI scanner. The ventral striatum is a putative reward region at the cross-roads of the mesolimbic dopamine and oxytocin systems (Skuse and Gallagher, 2009), and both dopamine and oxytocin are known to facilitate pair bonding in female prairie voles (Young et al, 2005). Although oxytocin was not measured during the scanning session, it is possible that the higher oxytocin levels characteristic of Type B mothers render stimuli associated with their own child more rewarding than is the case for Type A mothers, and this, in turn, could very well influence the mothers' responsiveness to her infant and hence the quality of attachment that develops.

A necessary caveat to these notable findings is that the authors measured oxytocin in plasma, but plasma oxytocin may not always be an accurate reflection of oxytocin levels in the brain (Landgraf and Neumann, 2004). An important next step would be to determine whether intranasal administration of oxytocin to Type A mothers before imaging augments their ventral striatum response to viewing pictures of their own infants. Nevertheless, this 
paper by Strathearn and colleagues is an important first step toward a better understanding of the biological basis of individual variation in mothering among women, a behavior sure to have consequences for the physical and mental development of the next generation.

\section{DISCLOSURE}

The author declares no conflict of interest.

\section{REFERENCES}

Berant E, Mikulincer M, Shaver PR (2008). Mothers' attachment style, their mental health, and their children's emotional vulnerabilities: a 7-year study of children with congenital heart disease. J Personality 76: 31-65.

Born J, Lange T, Kern W, McGregor GP, Bickel U, Fehm HL (2002). Sniffing neuropeptides: a transnasal approach to the human brain. Nat Neurosci 5: 514-516.
Ebstein RP, Israel S, Lerer E, Uzefovsky F, Shalev I, Gritsenko I et al (2009). Arginine vasopressin and oxytocin modulate human social behavior. Ann NY Acad Sci 1167: 87-102.

Heinrichs M, Domes G (2008). Neuropeptides and social behaviour: effects of oxytocin and vasopressin in humans. Prog Brain Res 170: 337-350.

Insel TR, Young LJ (2001). The neurobiology of attachment. Nat Rev Neurosci 2: 129-136.

Landgraf R, Neumann ID (2004). Vasopressin and oxytocin release within the brain: a dynamic concept of multiple and variable modes of neuropeptide communication. Front Neuroendocrinol 25: 150-176.

Skuse DH, Gallagher L (2009). Dopaminergic-neuropeptide interactions in the social brain. Trends Cogn Sci 13: 27-35.

van Ijzendoorn MH, Juffer F, Duyvesteyn MGC (1995). Breaking the intergenerational cycle of insecure attachment: a review of the effects of attachment-based interventions on maternal sensitivity and infant security. J Child Psychol Psychiatry 36: 225-248.

Young LJ, Murphy Young AZ, Hammock EA (2005). Anatomy and neurochemistry of the pair bond. J Comp Neurol 493: 51-57.

See related article by Strathearn et al on page 2655 . 